\title{
Septic Chronic Multifocal Osteomyelitis in Children: A Challenging Presentation of Osteomylitis in a Low Income Country
}

\author{
S. A. Kissou1, P. W. H. Dakouréé2, J. M. Somé3, M. Soulama², S. Coulibaly², M. Diallo² \\ ${ }^{1}$ Department of Pediatrics, Nazi BONI University, Bobo-Dioulasso, Burkina Faso \\ ${ }^{2}$ Department of Orthopaedics and Trauma, Nazi BONI University, Bobo-Dioulasso, Burkina Faso \\ ${ }^{3}$ Department of Medical Imaging, University Teaching Hospital, Ouahigouya, Burkina Faso \\ Email: *patdakoure@yahoo.fr
}

How to cite this paper: Kissou, S.A. Dakouré, P.W.H., Somé, J.M., Soulama, M., Coulibaly, S. and Diallo, M. (2018) Septic Chronic Multifocal Osteomyelitis in Children: A Challenging Presentation of Osteomylitis in a Low Income Country. Open Journal of Orthopedics, 8, 213-220.

https://doi.org/10.4236/ojo.2018.86024

Received: April 9, 2018

Accepted: June 1, 2018

Published: June 4, 2018

Copyright $\odot 2018$ by authors and Scientific Research Publishing Inc. This work is licensed under the Creative Commons Attribution International License (CC BY 4.0).

http://creativecommons.org/licenses/by/4.0/

\begin{abstract}
Background: The aim of this study was to describe the clinical, biological and x-ray presentation of Septic Chronic Multifocal Osteomyelitis (SCMO) and discuss the therapeutic difficulties of this pathology in an African Sub-Saharan teaching hospital. Patients and Methods: A retrospective study was conducted in the Orthopedics and Trauma department of the university teaching hospital of Bobo-Dioulasso (Burkina Faso). Over a period of two years, we selected all cases of chronic osteomyelitis. We studied the epidemiological, diagnostic and therapeutic aspects of SCMO cases. Results: Eleven cases of SCMO were identified. The mean age of the patients was 11.8 years and the sex ratio was 1.75 . There were 7 rural patients. The mean time to visit the hospital was 158 days. The most common reasons for consultation were pain (10 cases), swelling ( 9 cases), and fever ( 7 cases). Two were sickle cell patients. The most affected bones were the femur ( 10 cases), the tibia ( 9 cases) and the fibula (6 cases). The most frequent specific radiological lesions were sequestra (6 cases) followed by pandiaphysitis. Staphylococcus aureus was the most common aetiology. Treatment combined antibiotherapy and surgery (sequestrectomy and/or bone curettage and/or fistulectomy). Hip dislocations, pathological fractures and bone defect complicated the course of this disease. The therapeutic results was good in 3 patients and bad in 5 patients; the other 3 patients were lost to follow-up. Conclusion: Septic chronic multifocal osteomyelitis is a rare but formidable form of osteomyelitis in children. Therapeutic outcomes are often poor in hospitals with limited resources. The best strategy is prevention through early diagnosis and aggressive treatment of acute osteomyelitis.
\end{abstract}




\section{Keywords}

Bacterial, Children, Hematogen, Multifocal, Osteomyelitis

\section{Introduction}

Bone infections are common in children and result in a large number of cases in a chronic and disabling evolution in developing countries [1]. The frequency of bone infections and their habitual progression towards chronicity in SubSaharan Africa are related to several factors. Malnutrition and sickle cell disease prevalent in sub-Saharan Africa are the cause of the precarious immunity of many children in this part of the world. In addition, the precariousness of the technical platform (laboratory breakdown, absence of CT and MRI, few or no consultants) of public hospitals makes it difficult to take care of these patients. Thus, one of the most common forms of bone infection in children in tropical environments is chronic osteomyelitis [1] [2] [3]. This pathology constitutes a real social scourge because of the mediocre therapeutic results [2].

The location in the chronic osteomyelitis is in the majority of cases isolated but in certain situations it can concern several bones at once [3]-[8]. In fact, during a bacteriemia, the pathogenic germs can attach simultaneously to several bones and cause a multifocal acute hematogenous bone infection which can evolve towards chronicity [8]. This fearsome multifocal form of osteomyelitisis poorly described in the literature as cases reports [4] [5] [6] [7]. A perfect knowledge of these septic chronic multifocal osteomyelitis (SCMO) could allow a better management.

The aim of this study was to describe the clinical, biological and x-ray presentation of SCMO and discuss the therapeutic difficulties of this pathology in an African Sub-Saharian teaching hospital.

\section{Patients and Methods}

This retrospective and descriptive study was conducted in both pediatric and orthopedicand trauma departments of Bobo-Dioulasso university hospital, Burkina Faso (West Africa). Over a period of two years (2013-2015) we saw in our consultation 90 children for chronic hematogenous osteomyelitis. Among these patients, we selected for our study those who were less than 15 years old and who had a multiple localization of osteomyelitis (concomitant involvement of at least two different bones).

The studied parameters were collected from patients clinical records:

- epidemiological (age, gender, residency),

- clinical (reason for consultation, duration of evolution),

- radiographic (number of foci, localization, radiological lesions),

- biological (blood tests, bacteriology, electrophoresis, HIV test),

- therapeutic and outcome. 
Biological data (bacteriology and electrophoresis of hemoglobin) were not always available. We defined as good therapeutic results all the cases whose fistula disappeared (for the forms with fistulas), and/or regressions of the radiographic lesions after 4 months. All other results were considered bad.

\section{Results}

\subsection{Epidemiological Data}

During the study period, 11 cases of SCMO were identified, representing 12.2\% of all 90 chronic osteomyelitis cases in children. The average age was 11.8 years with extremes of 6 and 14 years. Seven patients were male and the sex ratio was 1.75. The majority of patients came from rural areas (seven out of eleven patients).

\subsection{Clinical Data}

The most common reasons for consultation were pain, swelling, and fever. The average duration of evolution was 158 days [10 - 575 days].

\subsection{Biological Data}

Two patients have sickle cell anemia and HIV serology was negative in all patients. Infectious status included hemogram, sedimentation rate and C-reactive Protein (CRP).The mains results were as follows:

- Anemia in all patients with 2 severs cases (hemoglobin $<6 \mathrm{~g} / \mathrm{dl}$ ),

- leukocytosis (6 cases) and 1 case of leukopenia;

- High rate of sedimentation rate in all patients;

- CRP was elevated in all cases.

The search for the pathogen was positive in seven patients. In five cases one organism was isolated. In the two other cases, it was a poly-infection. The aetiological micro-organisms were Staphylococcus aureus $(\mathrm{n}=5)$, Escherichia coli $(\mathrm{n}$ $=1)$, Pseudomonas aeroginosa $(\mathrm{n}=1)$, Proteus mirabilis $(\mathrm{n}=1)$, Streptococcus pneumonia $(\mathrm{n}=1)$, Klebsiella pneumonia $(\mathrm{n}=1)$. As for the antimicrobial susceptibility, resistance was frequently found for Penicillin A, Oxacillin, Amoxicillin + Clavulanic acid and Cotrimoxazole.

\subsection{Radiographic Aspects}

We counted a total of 33 foci with an average of 3 foci per patient (Table 1). We had noted in one patient up to five osteomyelitis foci.

The most frequent radiographic lesions were sequestrum and pandiaphysitis. There were 2 cases of hip dislocation and one trochanteric fracture complicating osteomyelitis (Figure 1).

\subsection{Treatment}

Medical treatment consisted of antibiotic administration to all patients (double in 9 cases and triple in two cases). The most commonly used drugs based on 


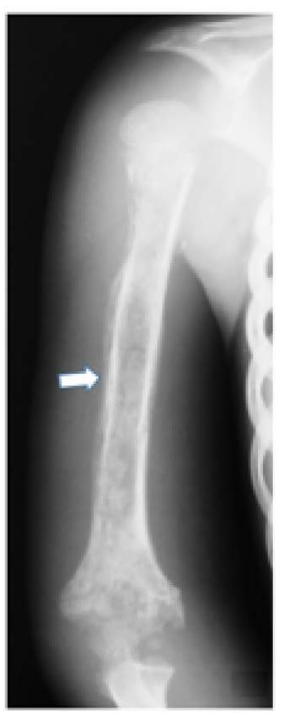

(a)

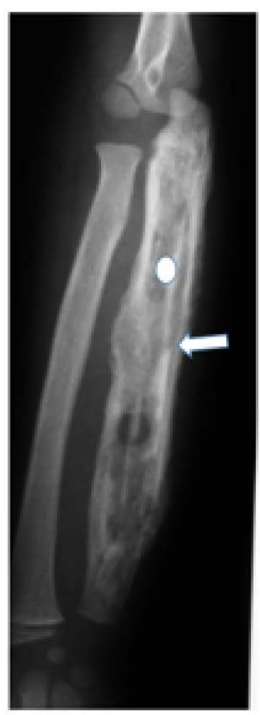

(b)

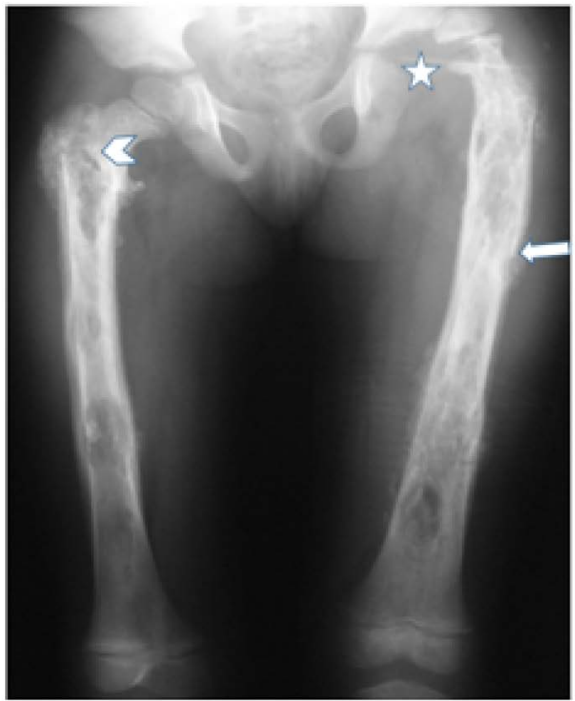

(c)

Figure 1. X-ray photographs of a patient with Septic Chronic Multifocal Osteomyelitis (humerus, ulna and both femurs). Pandiaphysitis (arrows) and sequestrum (round) were the most frequents lesions. Hip dislocation (star) and fracture (arrow head) were osteo-articular complications.

Table 1. Lesions distribution by location.

\begin{tabular}{cc}
\hline Location & Frequency \\
\hline Upper limb & 5 \\
Humerus & 2 \\
Ulna & 2 \\
Radius & 1 \\
Lower limb & 27 \\
Femur & 10 \\
Tibia & 9 \\
Fibula & 6 \\
Toes's phalanges & 1 \\
Calcaneus & 1 \\
Pelvic girdle & 1 \\
Hip bone & 1 \\
Total & 33 \\
\hline
\end{tabular}

bacteria sensitivity were Lincomycin, Gentamicine, Ceftriaxone, and Ciprofloxacin. The mean duration of the treatment was 69 days.

Plaster of Paris was used for the limbs stabilization in all cases. Surgery was performed in seven cases and consisted of sequestrectomy, bone curettage and fistulectomy. Sequestrectomy caused multiple bone loss in five cases (Figure 2(b)). 

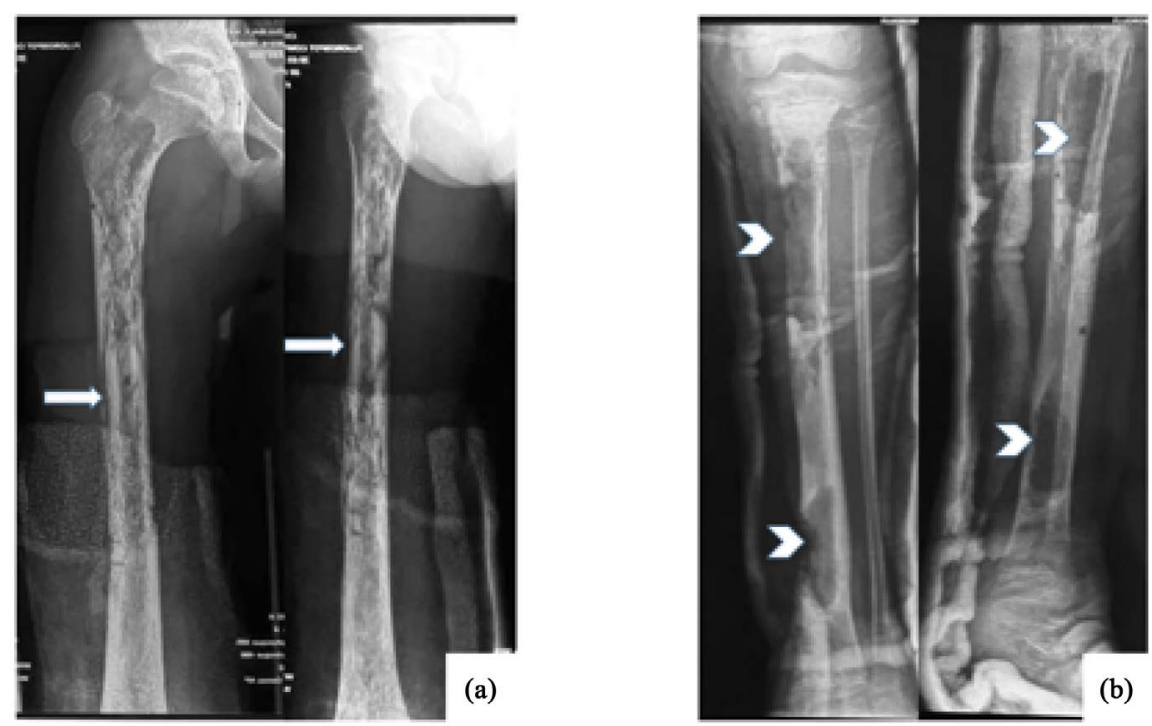

Figure 2. Photographs of tight (a) and leg (b) X-ray of a 9 years old boy with mutifocal osteomyelitis (right femur and ipsilateral tibia). Pandiaphisitis and sequestrum (arrows) was the lesion on the femur. Staged bone loss on the tibia (arrow heads).

\subsection{Outcomes}

The average hospital stay was 32.5 days (range 6 to 118 days). Two patients was lost of follow-up. Tree patients was successfully treated. An unfavorable result was observed in five cases.

\section{Discussion}

SCMO is the localization of chronic osteomyelitis at several bone in the same patient [3]-[8]. It is the evolution towards chronicity of at least two haematogenous osteomyelitic foci of concomitant appearance on two or more different bones. Thus, it is a condition that results from prolonged osteomyelitis complicating the development of acute osteomyelitis or chronic osteomyelitis, which is considered to be a complicated form of acute osteomyelitis by inadequate antibiotic therapy [8] [9].

SCMO is an infrequent disease; indeed, it represents $9.5 \%$ to $16 \%$ of chronic osteomyelitis cases [3] [9]. Unlike the acute forms reported by Sreenivas [8], the chronic form that we describe is a disease of big child and adolescent (average age: 11.8 years). It occurs mostly in children from rural setting. Sickle cell anemia and malnutrition (which is common is rural population in our country) certainly explain chronic anemia responsible for decreased immunity, leading to multifocal bone involvement [6] [7] [8] [10].

Apart the concomitant involvement of several bones, the clinical symptomatology is that of a classical chronic osteomyelitis. In our study we noted up to five different foci in the same patient; the average of foci being three. Thus, patients present with multiple fistulae on several limb segments; the lower limb being the seat of predilection [4] [7]. Diagnosis is confirmed on plain X-ray and Biology. Plain X-ray show evidence of severe chronic bone infection (large se- 
questra and pandiaphysitis) reaching several bony pieces at a time. Osteoarticular complications may occur. Indeed, we observed 2 cases of hip dislocations. Involvement of intracapsular metaphyses such as that of the proximal femur, often resulting in septic arthritis [11] that can be complicated by septic hip dislocation as in our series (Figure 1).

Samples of pus in pre or intraoperative identify the micro-organism responsible and test its sensitivity to antibiotics. The most frequently isolated germs was Staphylococcus aureus and gram-negative micro-organisms [5] [6] [8]. These micro-organisms were mostly resistant to usual antibiotics making this infection difficult to treat.

SCMO must be differentiated from recurrent multifocal chronic osteomyelitis (RCMO), which is a non-infectious inflammatory pathology that appears to be genetic [12]. RCMO is a rare disease that affects children. It manifests as multifocal bone lesions that mimic chronic pyogenic osteomyelitis. Differential diagnosis is done by laboratory tests. Indeed, RCMO is characterized by a biological inflammatory syndrome and sterility of bacteriological samples [12]. Therapeutically, there is no need for antibiotics but rather anti-inflammatory and bisphosphonates. In addition, the evolution of this pathology is marked by the disappearance of symptoms in a few years, most often without sequelae [12].

Management of SCMO is a challenge for the medical team. Due to the complexity of the disease, care must be multidisciplinary, involving clinicians, radiologists and biologists [6]. Indeed, faced with an SCMO, several problems must be taken into account. The virulence of germs, their resistance to usual antibiotics and the bone sclerosis (making the bone impermeable to antibiotics) make treatment difficult [11]. In addition to these factors, host deficiencies such as sickle cell anemia (which have a notable susceptibility to skeletal infection)will increase management difficulties [5] [6] [10].

The characteristic surgical complications of SCMO is multiple bone defects (Figure 2) which is a real tragedy in limited resource setting like our hospital. In fact, these bone defects require reconstruction techniques that are not always available in Africa [10] [13] [14].

Despite a good treatment, evolution is not always favorable [7] [8]. It is often marked by the persistence of fistulas or the recurrence after drying up of the infection.

This study is a preliminary report on SCOM. Because of its retrospective nature, the small number of patients and the poverty of the diagnostic and therapeutic means, the results of this study do not allow us to draw definite conclusions on the pathophysiology, the favoring factors and the best therapeutic strategy of SCOM. A prospective study on a larger number of cases will better answer these questions

\section{Conclusion}

Septic chronic multifocal osteomyelitis is a rare but formidable form of osteo- 
myelitis in children. Therapeutic outcomes are often poor in hospitals with limited resources. The best strategy is prevention through early diagnosis and aggressive treatment of acute osteomyelitis.

\section{References}

[1] Jones, W.H., Beckles, V.L.L., Akinola, B., Stevenson, A.J. and Harrison, W.J. (2011) Chronic Haematogenous Osteomyelitis in Children. An Unsolved Problem. The Bone \& Joint Journal (Br), 93-B, 1005-1010. https://doi.org/10.1302/0301-620X.93B8.25951

[2] Museru, L.M. and Mcharo, C.N. (2001) Chronic Osteomyelitis: A Countinuing Orthopaedic Challenge in Developing Countries. International Orthopaedics (SICOT), 25, 127-131. https://doi.org/10.1007/s002640100239

[3] Nacoulma, S.I., Ouédraogo, D.-D., Nacoulma, E.W.C., Korsaga, A. and Drabo, J.Y. (2007) Ostéomyélites chroniques au CHU de Ouagadougou (Burkina Faso). Etude rétrospective de 102 cas (1996-2000). Bulletin De La Societe De Pathologie Exotique, 100, 264-268.

[4] Delgado-Castro, M., Rodriguez, M., Guitian, R., Marquez, R., Dominguez, M.J. and Naval, P. (2002) Chronic Infections Multifocal Osteomyelitis vs Chronic Recurrent Multifocal Osteomyelitis: With Reference to One Case. Revista Española de Medicina Nuclear e Imagen Molecular, 21, 356-361. https://doi.org/10.1016/S0212-6982(02)72107-1

[5] Cabaret, B., Couëc, M.L., Lorrot, M., Launay, E. and Gras-LeGuen, C. (2013) Infection osteoarticulaire multifocale à Salmonella non typhi chez une enfant drépanocytaire. Archives de Pédiatrie, 20, 398-402.

https://doi.org/10.1016/j.arcped.2013.01.019

[6] Olaniyi, J.A., Alagbe, A.E., Olutoogun, T.A. and Busari, O.E. (2012) Multiple Bone and Joint Diseases in a Nigerian Sickel Cell Anemia: A Case Report. Mediterranean Journal of Hematology and Infectious Diseases, 4, e2012023.

https://doi.org/10.4084/mjhid.2012.023

[7] Bachmeyer, C., Lionnet, F., Gibeault, M. and Damsin, J.-P. (2008) Chronic Multifocal Osteomyelitis due to Clostridium Difficile in an Sickle Cell Anemia. The Pediatric Infectious Disease Journal, 27, 950-952. https://doi.org/10.1097/INF.0b013e318185a068

[8] Sreenivas, T., Nataraj, A.R., Menon, J. and Patro, D.K. (2011) Acute Multifocal Haematogenous Osteomyelitis in Children. Journal of Children's Orthopaedics, 5, 231-235. https://doi.org/10.1007/s11832-011-0347-1

[9] Labbé, J.-L., Peres, O., Leclair, O., Goulon, R., Scemama, P., Jourdel, F., Menager, C., Duparc, B. and Lacassin, F. (2010) Acute Osteomyelitis in children: The Pathogenesis Revisited? Orthopaedics \& Traumatology: Surgery \& Research, 96, 268-275. https://doi.org/10.1016/j.otsr.2009.12.012

[10] Diémé, C., Sarr, L., Guèye, A.B., Coulibaly, N.F., Sané, A., Ndiaye, A. and Sèye, S. (2014) Therapeutic Aspects of Chronic Bone Infections and Management Challenges. Open Journal of Orthopedics, 4, 21-26. https://doi.org/10.4236/ojo.2014.42004

[11] Calhoun, J.H., Manring, M.M. and Shirtliff, M. (2009) Osteomyelitis of the Long Bones. Seminars in Plastic Surgery, 23, 59-72. https://doi.org/10.1055/s-0029-1214158

[12] Ferguson, P.J. and Sandu, M. (2012) Current Understanding of the Pathogenesis and Management of Chronic Recurrent Multifocal Osteomyelitis. Current Rheu- 
matology Reports, 14, 130-141. https://doi.org/10.1007/s11926-012-0239-5

[13] Wirbel, R. and Hermans, K. (2014) Surgical Treatment of Chronic Osteomyelitis in Children Admitted from Developing Countries. African Journal of Paediatric Surgery, 11, 297-303. https://doi.org/10.4103/0189-6725.143133

[14] Geurts, J., Hohnen, A., Vranken, T. and Moh, P. (2017) Treatment Strategies for Chronic Osteomyelitis in Low- and Middle-Income Countries: Systematic Review. Tropical Medicine \& International Health, 22, 1054-1062.

https://doi.org/10.1111/tmi.12921 